\title{
Adaptive function and brain evolution
}

\author{
Fernando Martínez-García ${ }^{1}$, Luis Puelles ${ }^{2}$, Hans J. Ten Donkelaar ${ }^{3}$ and Agustín González ${ }^{4}$ \\ Laboratori de Neuroanatomia Funcional Comparada, Departament de Biologia Funcional, Universitat de València, Burjassot (Valencia), Spain \\ 2 Departamento de Ciencias Morfológicas y Psicobiología, Universidad de Murcia, Murcia, Spain \\ ${ }^{3}$ Radboud University Nijmegen Medical Center, Nijmegen, Netherlands \\ ${ }^{4}$ Department of Cell Biology, Universidad Complutense de Madrid, Madrid, Spain \\ *Correspondence: fernando.mtnez-garcia@uv.es
}

Comparing brains is not a mere intellectual exercise but also helps to understand how the brain enables adaptive behavioral strategies to cope with an ever-changing world and how this complex organ has evolved during the phylogeny. For instance, comparative neurobiology helps understanding the specific features of our species, an issue that attracted scientists since the time of Santiago Ramon y Cajal. Following this tradition, 20 years ago Hans ten Donkelaar and Gerhard Roth started the European Conferences on Comparative Neurobiology (ECCN). This e-book includes some of the contributions to the last meeting, the sixth ECCN (Valencia, Spain; April 22-24 2010), plus selected works by several authors interested in the topic. The 7th ECCN Meeting will be organized by Andras Csillag and held in April 2013 in Budapest (Hungary).

One of the tenets of evolutionary biology is that evolution relays on development: developmental changes result in anatomo-functional modifications that may eventually be selected. In their chapter, Charvet and Striedter explore this idea in birds, by comparing forebrain development in precocial species not showing learned vocalizations with parrots and songbirds, altricial birds with learned vocalizations. As compared to precocial birds, altricial ones display a delayed neurogenesis thus suggesting that this developmental modification boosts infant learning capacities, a phenomenon arguably applicable to human evolution. This same issue is also tackled in this book by Matsunaga and Okanoya, who compare the expression of cadherins (molecules involved in cell-cell interactions related to various aspects of development; Hirano et al., 2003) in vocal, auditory, and visual centers of the brain of vocal learners and non-learner birds. As expected, cadherin expression shows a much higher variability in vocal and auditory than in visual areas between learners and non-learners.

Evolution of the brainstem has been fairly conservative. Consequently, a comparative analysis of its development might be useful in understanding the specific adaptations it has undergone through phylogeny. In his chapter, Nieuwenhuys has used a topologyguided projection procedure to elaborate a bidimensional map of the brainstem that has proven very useful for this kind of comparative studies. A complementary strategy is used by Rodríguez-Moldes and co-authors: by analysing the expression of morphogenetic genes, they are able to compare specific neuronal populations in the brainstem of different vertebrates. This strategy is especially helpful to understand the comparative neuroanatomy of highly variable structures. For instance, Wullimann and collaborators apply it to compare the rhombic lip derivatives of fish and tetrapods, thus revealing general commonalities in cerebellar organization.

This approach has promoted an actual revolution in comparative neurobiology. Analysis of gene expression patterns using a correct view of the anteroposterior axis of the neural tube led Puelles and
Rubenstein (2003) to define three neuromeres in the forebrain, the prosomeres, plus a secondary (apparently not divided) prosencephalon (hypothalamus, retinae, and telencephalon). Merchan and co-authors propose the term genoarchitecture to define the analysis of the architecture of a neural center on the basis of its pattern of gene expression. As an example, they report a fine-grained analysis of the genoarchitecture of the avian pretectum (alar plate of prosomere 1), which very likely fits the pretectum of other vertebrates.

Genoarchitecture is currently being used to understand the complex organization of the secondary prosencephalon. For instance, Morales-Delgado et al. report the expression of morphogenetic genes in mouse embryos. Their findings reveal two major anteroposterior divisions in the hypothalamus (prosomeres 4 and 5?), each one consisting of alar, basal, and floor plates, in which tangential migrations contribute to the structural complexity of the adult hypothalamus. Through a genoarchitectonic comparative analysis of the hypothalamus and the non-evaginated telencephalon (preoptic region), Moreno and Gonzalez are able to identify some of the fundamental changes that occurred in the agnathangnathostome and anamniote-amniote transitions. In the same line, Dominguez et al. have found that in the amphibian forebrain the expression of the morphogetic gene $N k \times 2.2$ neatly delineates the alar/basal boundary. In contrast to mammals and birds, however, this gene is not expressed in the amphibian basal telencephalon, which might explain differences in the organization of the cerebral vesicles between amniotes and anamniotes.

Some species occupy a crucial position in the lineage of vertebrates, making their brains especially interesting from a comparative viewpoint. For instance, lampreys and hagfishes (agnathans, jawless vertebrates) display a rudimentary telencephalon, whose comparative significance, in particular their pallium, is still controversial. Whereas in gnathostomes (jawed vertebrates) GABAergic cells reach the pallium after tangential migration from the ganglionic eminences (Marín and Rubenstein, 2003), the apparent lack of a medial ganglionic eminence in the lamprey brain (Kano et al., 2010) raises doubts about the origin of the agnathan pallial GABAergic cells. In their contribution, Pombal et al. tackle this issue by analysing the development and adult distribution of GABAergic cells in the cerebral hemispheres of lampreys. On the other hand, Northcutt and González report a modern interpretation of the telencephalon of the coelacanth, the only living representative of a sister group of the tetrapods and of lungfishes. This constitutes an extraordinary opportunity for understanding the evolutionary history of the cerebral hemispheres in vertebrates.

The evolutionary origin of the six-layered neocortex is one of the preferred topics of comparative neuroanatomy. Dealing with it, Shepherd proposes a common cortical microcircuit in the cortices of 
mammals (isocortex, hippocampus, olfactory cortex) and non-mammals (dorsal cortex of turtles) in which connections among pyramidal cells, direct and indirect (via non-pyramidal interneurons), would mediate forward inhibition, recurrent inhibition, recurrent excitation, and lateral inhibition. Additive modifications to this scheme would explain the appearance of the sophisticated isocortex from the simpler dorsal (general) cortex of ancestral reptiles. In their review, Montiel and co-workers discuss the putative role of the cortical subplate in establishing the connections of this canonical cortical microcircuitry, and its possible role in the evolutionary transition from the three-layered to the complex six-layered cortex.

Pyramidal cells show a huge structural diversity among different cortical areas and among species. The detailed morphometric analysis of the dendritic tree of pyramidal cells in different cortical areas of three cercopithecid primates performed by Elston et al. reveals significant interspecies differences in prefrontal areas, with important interindividual variation in all three species. In contrast, sensory, motor, or cingulate cortices show less variability. This constitutes a paradigmatic case of the relationship between form and function: the complexity of the dendritic arborization of pyramidal cells reflects the capacities in planning, prioritizing, and conceptualization of the different primate species, including humans.

The adaptive function of brain systems is another current topic of comparative neurobiology. The study of the evolution of a given function or functional system becomes, therefore, an interesting issue. For instance, the cladistic analysis of the evolution of the vomeronasal system performed by Ubeda-Bañon and co-authors indicates that ancestral vertebrates showed two chemosensory systems, olfactory and vomeronasal, with different receptors, primary and secondary projection areas. Specific evolutionary pressure (e.g., return to aquatic life, flight, or bipedalism) might have resulted in the involution of the vomeronasal system in some taxa. The putative role of the vomeronasal system in the detection of pheromones and other chemical signals makes this issue very interesting to evaluate current ideas on pheromonal communication in humans.

Unlike other sensory systems, vision has a mobile sensory organ (the eye) whose position and orientation determines perception. Consequently, an oculomotor function coordinated with neck-body movements is crucial for vision. In their chapter Luque and collaborators study the GABAergic control of oculomotor neurons in fish and compare it with the mammalian pattern. Despite the enormous differences in the structure of the brain, body, and eyes, fish and mammals share similar neural mechanisms for vestibulo-oculomotor reflexes and higher level gaze control, probably developed early in vertebrate evolution, as soon as two mobile camera eyes appeared.

Comparative neurobiology is also useful for studying the neural basis of complex behaviors. An interesting case is the social grouping. Using five species of estrildid finches (closely related species with similar behaviors in other respects) that differ in group size from highly gregarious to territorial/asocial, Goodson and Kingsbury show that non-apeptidergic systems encode the valence of social stimuli (e.g., songs and visual displays in most birds) by modulating motivation and/or anxiety-like responses to them. Since nonapeptides are involved in basic social behaviors across a wide range of vertebrates, it is likely that they may be common or even ubiquitous targets of selection during social evolution.

In contrast to birds, in rodents and squamate reptiles sociosexual interactions are dominated by chemosensory stimuli, pheromones that elicit motivated behaviors (e.g., mate search, sexual behavior). Novejarque and co-authors have traced the connections between the olfactory and vomeronasal amygdala with specific portions of the ventral striato-pallidum, putatively conveying chemosensory information to the reward system of the brain. This is a well-conserved pathway. As suggested by Ubeda-Bañon et al. in their chapter, the "vomeronasal amygdala" of birds and primates might have been colonized by non-vomeronasal stimuli (olfactory, visual, and auditory) that would have acquired a preeminent role as signals for socio-sexual behaviors.

Motivated responses are among the most complex and adaptive behaviors in vertebrates. Dopamine is a neuromodulator with a key role in motivation, but also participates in other functions (sensory processing, neuroendocrine, learning) distributed in several anatomical areas. This suggests that the brain of vertebrates possess several independent dopaminergic systems (e.g., Smeets and Reiner, 1994) probably derived from a single system in the common ancestor of chordates. Yamamoto and Vernier perform a comprehensive comparative analysis of dopamine neurotransmission in vertebrates. By identifying the molecular machinery of dopamine synthesis and neuromodulation in different vertebrates, they characterize the pattern of differentiation of dopaminergic cells. This allows a better understanding of the physiology and pathology of dopamine systems, which nicely illustrates the strength of comparative neurobiology.

\section{REFERENCES}

Hirano, S., Suzuki, S. T., and Redies, C. (2003). The cadherin superfamily in neural development: diversity, function and interaction with other molecules. Front. Biosci. 8, d306-d355.

Kano, S., Xiao, J., Osório, J., Ekker, M., Hadzhiev, Y., Müller, F., Casane, D., Magdelenat, G., and Rétaux, S. (2010). Two lamprey hedgehog genes share non-coding regulatory sequences and expression patterns with gnathostome hedgehogs. PLoS ONE 5, e13332. doi: 10.1371/journal.pone.0013332

Marín, O., and Rubenstein, J. L. R. (2003). Cell migration in the forebrain. Annu. Rev. Neurosci. 26, 441-483.

Puelles, L., and Rubenstein, J. L. (2003). Forebrain gene expression domains and the evolving prosomeric model. Trends Neurosci. 26, 469-476.

Smeets, W. J. A. J., and Reiner, T. (1994). Phylogeny and Development of Catecholamine Systems in the CNS of Vertebrates. Cambridge: Cambridge University Press.

Received: 25 April 2012; accepted: 08 May 2012; published online: 29 May 2012. Citation: Martinez-Garcia F, Puelles L, Ten Donkelaar HJ and González A (2012) Adaptive function and brain evolution. Front. Neuroanat. 6:17. doi:10.3389/fnana.2012.00017 Copyright (c) 2012 Martinez-Garcia, Puelles, Ten Donkelaar and González. This is an open-access article distributed under the terms of the Creative Commons Attribution Non Commercial License, which permits non-commercial use, distribution, and reproduction in other forums, provided the original authors and source are credited. 